\title{
Industry 4.0 with Cyber-Physical Integration: A Design and Manufacture Perspective
}

\author{
Alfredo Alan Flores Saldivar ${ }^{1}$, Yun Li ${ }^{1}$, Wei-neng Chen ${ }^{2}$, Zhi-hui Zhan ${ }^{2}$, Jun Zhang ${ }^{2}$, Leo Yi Chen ${ }^{3}$ \\ ${ }^{1}$ School of Engineering, University of Glasgow, Oakfield Avenue, Glasgow G12 8LT, U.K. \\ (Email: a.flores-saldivar.1@research.gla.ac.uk; Yun.Li@glasgow.ac.uk) \\ ${ }^{2}$ School of Advanced Computing, Sun Yat-sen University, Guangzhou, China \\ ${ }^{3}$ School of Engineering and Build Environment, Glasgow Caledonian University, Glasgow, U.K.
}

\begin{abstract}
Foreseeing changes in the way companies manufacture products and provide services, future trends are emerging in design and manufacture. Together with growing internet applications and technologies connected through the cloud, a new Industrial Revolution, named "Industry 4.0", aims to integrate cyber-virtual and cyber-physical systems to aid smart manufacturing, as presented in this paper. Connecting information and physical machinery, this new paradigm relies on how effective and fast connectivity are achieved for Industry 4.0. A new generation of wireless connection, 5G, will help and accelerate this trend. Following analysis of the present cyberphysical integration for the $4^{\text {th }}$ Industrial Revolution, this paper also investigates future methodologies and trends in smart manufacturing, design and innovation.
\end{abstract}

Keywords- Cyber-physical integration; cyber-physical systems; Industry 4.0; smart manufacturing; networked autonomous production; CAD; CAutoD

\section{INTRODUCTION}

Design and manufacture are currently moving to a new paradigm, targeting innovation, lower costs, better responses to customer needs, optimal solutions, intelligent systems, and alternatives towards on-demand production. The concept that highlights this significant evolution is "Industry 4.0" (I4), dubbed the "4th Industrial Revolution" [1], with associated concepts of networked embedded systems, cyber-physical systems (CPS), smart factory, Internet of Things (IoT), Internet of Services (IoS) and "Internet+", to name but a few. All these trends have in common the integration of several features in the same place as a response to challenges of computerized decision making and big data that are proliferated by the internet and cloud computing (CC).

To gauge the development and trends, this paper aims to analyze cyber-physical integration for design and manufacture, and to present a timely survey on Industry 4.0. Section 2 set the scene of Industrial Revolutions (IRs), with cyber-physical systems detailed in Section III. Necessary information and communication technologies (ICT) are analyzed in Section IV. Conclusions are drawn and future agendas are discussed in Section V.

\section{INDUSTRY 4.0 - AN EVOLUTIONARY REVOLUTION}

\section{A. What Industry 4.0 Is}

Ever since the beginning of industrialization, technological advances have led to socio-economic paradigm shifts which are today termed "industrial revolutions", i.e., mechanization with steam power for the 1st IR $\rightarrow$ electrical energy for mass production in the 2nd IR $\rightarrow$ automated production with electronics and control in the 3rd IR. Today, with advances in digitalisation and the internet, "smart manufacturing" and "smart factories" are becoming a reality, where the manufacturing value chain in the physical world can be integrated with its virtual copy in the cyberspace through CSP and IoT, and then be seamlessly integrated with IoS. Tempted by these future expectations, the term "Industrie 4.0" or "Industry 4.0" was coined a priori by the German government promoting their "High-Tech Strategy 2020 Action Plan" in 2013 for a planned "4th industrial revolution" [2]-[4].

The terminologies "Smart Industry" and I4 describe the same technological evolution from the microprocessor embedded manufacturing systems to the emerging CPS, smartly linking (i) demand to (ii) manufacture, (iii) supply, and (iv) services by the internet. Via decentralising intelligence, object networking and independent process management interact with the virtual and real worlds, heralding a crucial new aspect of future industrial production process that integrates the above four processes. In short, I4 represents a paradigm shift from "centralised" to "decentralised" production, a reversal of the logic of production process thus far. The design principles of I4 components are shown in Table 1 [4].

Table 1 Design Principles of I4 Components

\begin{tabular}{|c|c|c|c|c|}
\hline Design & CPS & IoT & IoS & $\begin{array}{c}\text { Smart } \\
\text { Factory }\end{array}$ \\
\hline Interoperability & $\mathrm{X}$ & $\mathrm{X}$ & $\mathrm{X}$ & $\mathrm{X}$ \\
\hline Virtualisation & $\mathrm{X}$ & - & - & $\mathrm{X}$ \\
\hline Decentralisation & $\mathrm{X}$ & - & - & $\mathrm{X}$ \\
\hline Real-Time Capability & - & - & - & $\mathrm{X}$ \\
\hline Service Orientation & - & - & $\mathrm{X}$ & - \\
\hline Modularity & - & - & $\mathrm{X}$ & - \\
\hline
\end{tabular}

\section{B. Importance of the Strategised Industry Revolution}

The first three industrial revolutions came about as a result of centralization for production. Now, businesses will establish global networks that incorporate their machinery, warehousing systems and production facilities in the shape of a cyberphysical system, comprising "smart machines", storage systems and production facilities capable of autonomously exchanging information, triggering actions and controlling each other independently. 
These form a "smart factory" that allows individual customer requirements to be met, whilst efficiency obtained in automated production is maintained. This means that even oneoff items can be manufactured profitably. In Industry 4.0, dynamic business and engineering processes enable last-minute changes to production and offer the ability also to respond flexibly to disruptions and failures. End-to-end transparency is provided over the manufacturing process, also facilitating optimized design and decision-making. Further, Industry 4.0 will result in new ways of creating value and novel business models. In particular, it will provide start-ups and small businesses with the opportunity to develop and provide downstream services. To both developed and developing economies, I4 will reduce factory-floor requirements and help progress of humanity.

\section{CYBER-PHYSICAL INTEGRATION}

A CPS collaborates computational entities which are in intensive connections with their surrounding physical world and on-going processes, providing and using, at the same time, data-accessing and data-processing services available on the internet [5]. A cyber-physical production system relies on the newest and foreseeable further developments of computer science, ICT, and manufacturing science. Concepts like autonomous cars, robotic surgery, intelligent buildings, and implanted medical devices are just some of practical examples that have already emerged in Research and Developments (R\&D) [6]

\section{A. Design of a Cyber-Physical System}

Cyber space and virtual systems represented by ICT are now getting integrated with physical control and production systems. This integration is enabling compression of development cycles by reuse of existing methodologies, methods, models, tools and techniques, encapsulated in integrated and customized models and components that can be rapidly used in an innovative or creative design. The unique challenges in CPS integration emerge from the heterogeneity of components and interactions. This heterogeneity drives the need for modelling and analyzing cross-domain interactions among physical and computational and networking domains, which demands deep understanding of the effects of heterogeneous abstraction layers in the design flow [7].

Figure 1 illustrates a well-funded approach to cyberphysical integration to meet design principles, mainly proposed in [8]. It highlights that analysis is a key issue in current CPS developments, integrating various objects, design methods and tools, aspect-oriented development methods and tools, multidomain physical modelling methods and tools, and formal methods that address different aspects of the development process of CPS. Systems specification, modelling and design method integration involve many aspects of integration at different levels, including:

- Integration of the physical world dimension, communication dimension and computation dimension;

- Integrated object-oriented methodology, multi-domain methodology, aspect-oriented methodology and formal techniques;

- Integration of different design views;
- Integration of the methods used to specify and implement systems requirements;

- Integration of tools that support these methods;

- Integration of physical components and cyber components;

- Integration of different representations;

- Integration of the multiple specification fragments produced by applying these methods and tools; and

- Integration between informal specification methods and formal specification methods.

Model, Methodology and Tool Integration are detailed in the following sections. These aspects help investigate future directions and trends in Industry 4.0 .

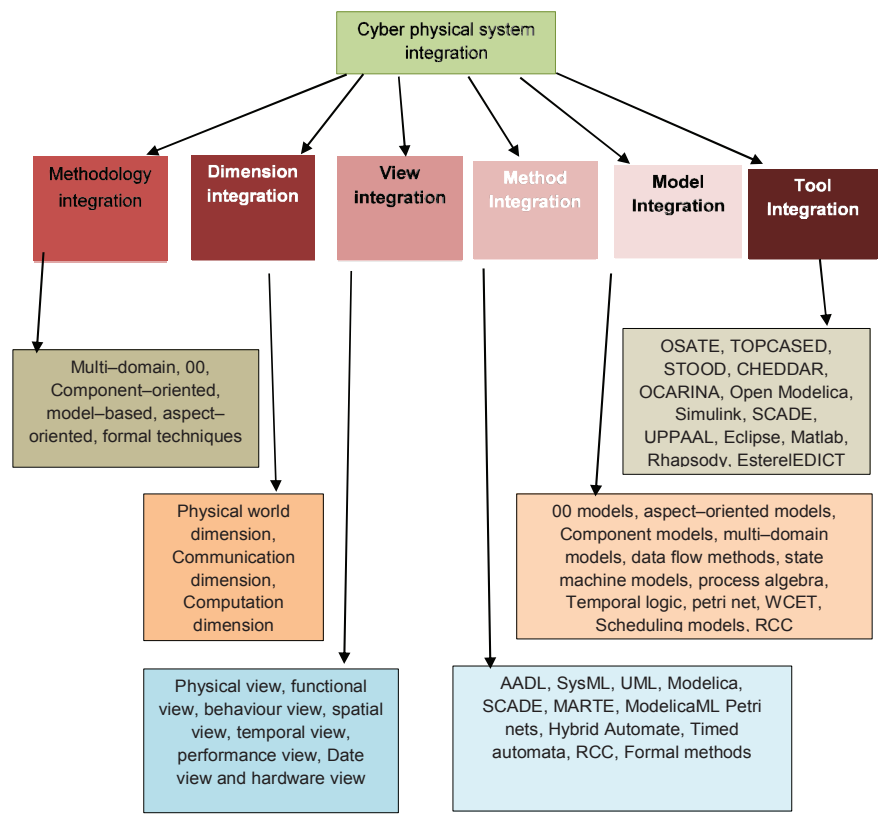

Figure 1. Integrated Approach to develop CPS.

\section{B. Model Integration for Manufacturing-Aware Design Flow}

As discussed, it is important to develop methodologies that integrate models, techniques, and tools that can be used in a design customized within its models and components. Components and models in a CPS are heterogeneous, spanning multiple disciplines (physical - thermal, mechanical, electrical, fluid,... and cyber - software, computing, cloud...). These require multiple models to represent the physical aspects, requirements, architectures, behaviours, spatial-temporal constraints, and interfaces, at multiple levels of abstractions [8].

Model and component-based design have been recognized as key technologies for radically changing productivity with CPS. Model-based design uses formal and sufficiently complete models, processes, their environments, and their interactions. The goal of a model-based design is "correct-by-construction", where properties of the synthesized models of the designed system predict the properties of the implemented or manufactured system with sufficient accuracy [9]. As a result, an integrated tool suite called OpenMETA has been developed to provide a manufacturing-aware design flow, which covers both cyber and physical design aspects. A new integration 
model for the OpenMETA suite was proposed in [9], as shown in Figure 2. Basically, the design flow is implemented as a multi-model composition/synthesis process that incrementally shapes and refines the design space using formal, manipulated models. It includes analysis and testing steps to validate and verify requirements and to guide the design process to achieve least complexity, and therefore the least risky and least expensive solutions. For example, the Adaptive Vehicle Make (AVM) [10] project, funded by the Defense Advanced Research Project Agency (DARPA), has constructed a fully integrated model and component-based design flow for the make process of a complex CPS.

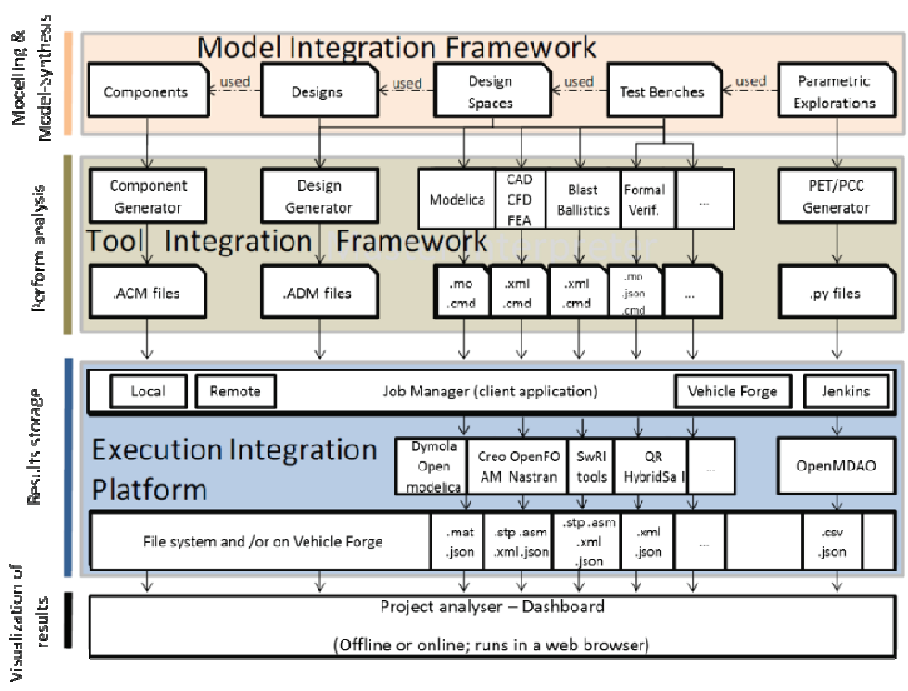

Figure 2. Model Integration: OpenMETA framework.

The main procedures of this design flow sketch the following phases:

1) Currently combinatorial or future intelligent design space exploration and architecture evaluation;

2) Behavioural design space exploration by progressively deepening from qualitative discrete behaviours to precisely formulated relational abstractions and to quantitative multi-physics, lumped parameter hybrid dynamic models using both deterministic and probabilistic approaches;

3) Geometric/structural design space exploration coupled with physics-based nonlinear finite element analysis of thermal, mechanical and mobility properties; and

4) Cyber design space exploration (both hardware and software) integrated with system dynamics.

As highlighted in Figure 2, these elements reflect the drive train challenge for the AVM development, emphasizing the $3 \mathrm{D} / \mathrm{CAD}$ tools and finite element analysis for verifying blast protection and hydrodynamic requirements remaining on the basic structure of the integration architecture. In synthesis, the modelling functions of the OpenMETA design flow are built on the following model types [9]:

1) AVM Component Models (ACM) with standard, composable interfaces;

2) Design Models (DM) that describe component architectures and related constraints;
3) Design Space Models (DSM) that define structural and architectural variabilities;

4) Test Bench Models (TBM) representing environment inputs, composed system models connected to a range of testing and verification tools for key performance parameters; and

5) Parametric Exploration Models (PEM) for specifying regions in the design space to be used for optimization and models for complex analysis flows producing results such as Probabilistic Certification of Correctness (PCC).

The first emphasis is placed on the development of a model integration language, so as to address heterogeneity to cover all relevant views of multi-physics and cyber domains and to achieve compositionality. Heterogeneity of the multi-physics, multi-abstraction and multi-fidelity design space, and the need for rapidly evolving/updating design flows require the use of a rich set of modelling languages usually influenced/determined by existing and emerging model-based design, verification and simulation technologies and tools. Consequently, the language suite and the related infrastructure cannot be static; it will continuously evolve. Then the second development is methodology integration in this framework [9]

\section{Method Integration}

To integrate modelling languages for CPS environments, mathematical models in this sense can bring together abstractions that are imported from individual languages and required for modelling cross-domain interactions. Proposed in [9], a language called CyPhyML is constructed as a lightweight, evolvable, composable integration language that is frequently updated and morphed. While these DSMLs may be individually quite complex (e.g., Modelica, Simulink, SystemC, etc...) ChyPhyML is relatively simple and easily evolvable. This "semantic interface" between CyPhyML and the domain specific modeling languages (DSML) shown in Figure 3 is formally defined, evolved as needed, and verified for essential properties (such as well-formedness and consistency) using the methods and tools of formal meta-modelling. By design, CyPhyML is moving in the opposite direction to unified system design languages, such as SySML or AADL. Its goal is specificity as opposed to generality, and heavy weight standardization is replaced by layered language architecture and specification of explicit semantics.

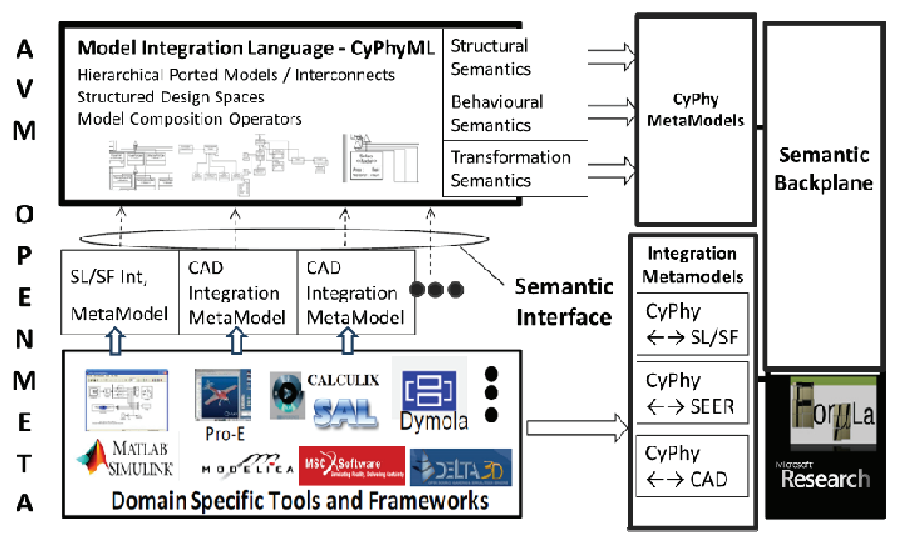

Figure 3. Method Integration Framework.

Mathematically precise formal semantics for model integration have been developed for introducing dynamic model 
integration. The key is to define the structural and behavioural semantics of the CyPhy model integration language using formal meta-modelling, a tool support formal framework for updating the CyPhy metamodels and verifying its overall consistency and completeness as the modeling languages evolving. In this case, the meta-modelling tool FORMULA from Microsoft Research can be used. This tool is sufficient for defining mathematically modelling domains, transformations across domains, as well as constraints over domains and transformations, since they are algebraic data types (ADTs) and constraint logic programming (CLP) based semantics.

In Figure 2, it is observed as part of the Model Integration that a large suite of modelling languages and tools for multiphysics, multi-abstraction and multi-fidelity modelling are included; OpenModelica, Dymola, Bond Graphs, Simulink/Stateflow, STEP, ESMOL and many others software that are useful for analysis. At the end CyPhyML model integration language provides the integration across this heterogeneous modelling space and the FORMULA - based Semantic Backplane provides the semantic integration for all OpenMETA composition tools [10] The next step in this case, will be to outline Tool Integration according to Development 3, in which execution integration is also provided as a whole platform.

\section{Tool Integration}

Using the same approaching to [9-11], in which the Tool Integration Framework of the OpenMETA incorporates a network of model transformations that include models for individual tools and integrate model-based design flows, model-transformations are used for the following roles:

1) Packaging: Models are translated into a different syntactic form without changing their semantics. Taking the development, AVM Component Models and AVM Design Models are translated into standard Design Data Packages (Figure 2, ACM and .ADM files) for consumption by a variety of design analysis, manufacturability analysis and repository tools.

2) Composition: Model- and component-based technologies are based on composing different design artefacts (such as DAE-s for representing lumped parameter dynamics as Modelica equations, input models for verification tools, CAD models of component assemblies, design space models, and many others) from appropriate models of components and component architectures.

3) Virtual prototyping: Several test and verification methods (such as Probabilistic Certificate of Correctness - PCC) require test benches that embed a virtual prototype of the designed system executing a mission scenario in some environment (as defined in the requirement documents). It is found that distributed, multi-model simulation platforms are the most scalable solution for these tests. The author selected the High Level Architecture (HLA) as the distributed simulation platform and integrated FMI Co-Simulation components with HLA.

4) Analysis flow: Parametric explorations of designs (PET), such as analyzing effects of structural parameters (e.g. length of vehicle) on vehicle performance, or deriving PCC for performance properties frequently require complex analysis flows that include a number of intermediate stages. Automating design space explorations require that Python files controlling the execution of these flows on the Multidisciplinary Design Analysis and Optimization (OpenMDAO6) platform (that are currently use in OpenMETA) are auto-generated from the test bench and parametric exploration models (Figure 2).

It is highlighted through this development, that both multiphysical and computation modelling present advantages choosing the specification of composition, i.e. for physical interactions; power flow oriented modelling (Modelica, Simscape or Bond Graph modelling languages) requires spending time typing for expressing and enforcing connectivity constraints achieving safe modelling of multi-physics interactions.

The OpenMeta model and tool integration technology needs and infrastructure for creating and executing complex analysis flows. Based on "software-as-a-service" aspect of this development, it allows end users (individuals, research groups, and large companies) to repositories, analytic services and design tools to lower the costs, and excluding the high costs of acquiring and maintaining desktop engineering tools. In Figure 4 is presented the platform for executing the part of tool integration, according to [9].

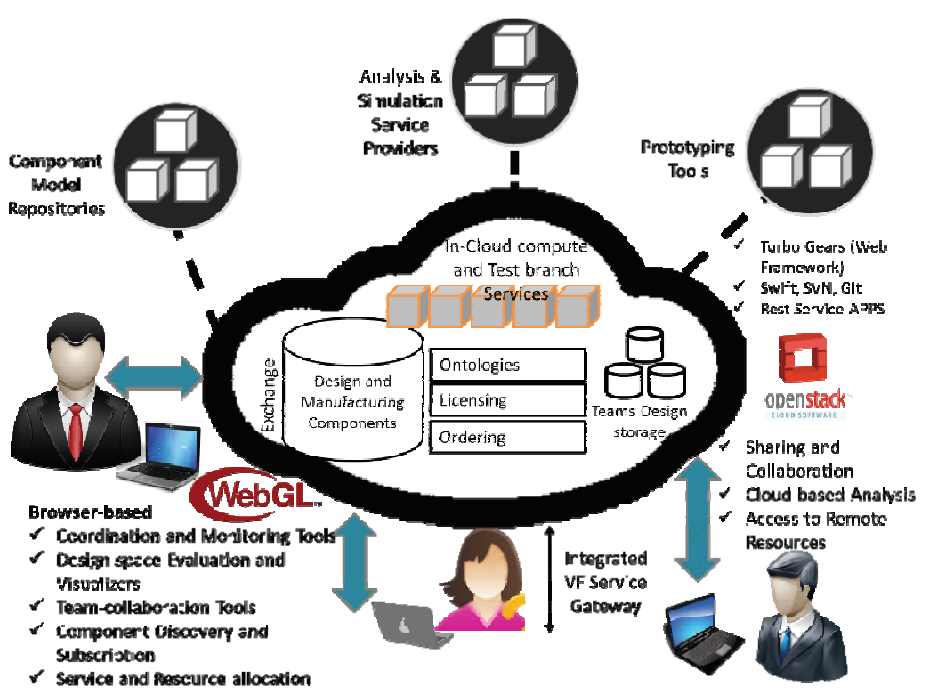

Figure 1. Tool Integration Framework.

The key fundamentals addressed in this platform are:

1) Resource elasticity and service staging, to address scalability and system wide optimization.

2) Managing the evolution of data, data presentations and their use by service and service integration over time.

With these fundamentals, it is clear that another matter need to be addressed for this platform, as shown in Figure 4. The evolution of data is key to better results and performance of this development.

\section{ICT INFRASTRUCTURE FOR INDUSTRY 4.0}

\section{A. Decentralized Computing}

One of the main barriers to Industry 4.0 is decentralized systems, as described in [12], for allocating hardware and software resources to individual workstation or desired 
location, which have been designed and operated with limited knowledge of the complete system. However, decentralized computing is now a trend in business environments. As there is no central decision maker, the information flow stays mostly local. Viewed as part of the control system, decentralization forms a self-organizing emergent system, with self-adaption, self-management, and self-diagnosis, keys in autonomic computing as well as Industry 4.0. This allows control of more complex systems, although global optimal performance cannot be guaranteed as firmly as with global controllers [12].

For implementing Smart Manufacturing schemes, ICT known as unified communications and integration of telecommunications, computers, necessary software, storage, and all those interfaces that allow users within the systems to access, store, transmit and manipulate information, must be addressed as well. As the number of users increases and the cloud computing grows in scale, decentralised cloud solution can play an importance role of infrastructure [13]. Decentralised intelligence can keep information and communication between the system components. By simulation and virtual design, manufacturing can be improved using optimization and control tools to set system scenarios. However, distributed optimization, owing to explosion in size and complexity of modern datasets, comprises the importance of solving problems and analysis.

Networked production leading to what Industry 4.0 is aiming to achieve, constantly collects Big Data sets, those data sets possess characteristics of being extremely large, highdimensional and data stored or collected in a distributed manner. All those characteristics can also be noted as part of the machine learning process. As a result, it has become of central importance to develop algorithms that are both rich enough to capture the complexity of modern data, and scalable enough to process huge datasets in a parallelized or fully decentralized fashion [14].

\section{B. Cloud Computing}

Through resources virtualization, cloud computing provides infrastructure, platform and software as services. "Cloud computing is a model for enabling ubiquitous, convenient, on demand network access to a shared pool of configurable computing resources (e.g. networks, servers, storage, applications, and services) that can be rapidly provisioned and released with minimal management effort or service provider interaction" [6]. This cloud model is composed of five essential characteristics, three service models, and four deployment models for optimal scheduling of resources [15].

For an I4 environment, private clouds, where data are restricted to the usage of a company, are imperative as a service linking between the private networks and the public ones, with a well-funded policy of data sharing and data accessing. The linkage between cloud computing and automation systems is strong. Nowadays, developments on both sides are getting involved, so as to achieve higher efficiency with less effort. With cloud computing, CPS are being optimized to reach manufacturing by the press of one button [16]. The gathering of knowledge and perspectives with the proposal of architecture for Global Information are showed in Figure 5.

Facing this paradigm, the integration of CPS, IoT, IoS and $\mathrm{CC}$ leads to a transformation, more than a revolution. Those subjects already mentioned before comprise the trend and need to implement Industry 4.0, which gives the way of reorganizing the modes of production using existing tools and placing reliance on networks. With the mergence between the Internet and factories, Industry 4.0 is characterized by the constant communication and linkage among production, supply chain, tools and workstations via the Internet and virtual networks.

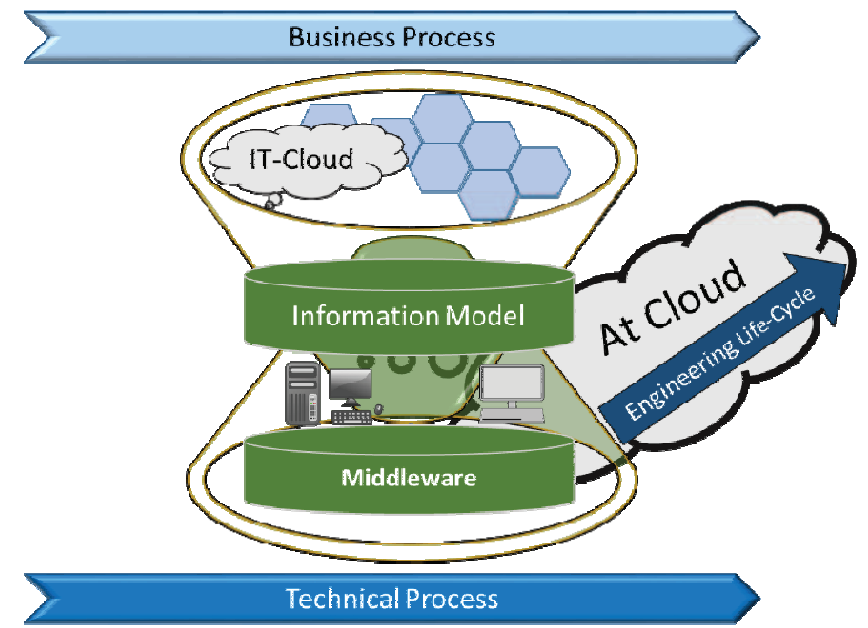

Figure 2. Architecture for Global Information with use of Cloud Computing.

\section{Model-Based Integration}

Considering Integration as part of the whole-system automation, it comes another topic of interest: "embeddedautomation systems", those which refer to control systems encompassing both domains (control-dominated and datadominated) and which can be designed using one unified approach. This approach was proposed in [17], where the control-dominated parts deal with asynchronous inputs from an external environment, while the data-dominated parts process these events by calling appropriate functions.

While trying to reduce IT costs, improve efficiency of sharing processes and enhance scalability inside companies' data and applications integration, there are certain levels of integrations known as: presentation level integration, business process integration (Service Oriented Architecture), data integration and communications-level integration. Depending on a company's particular needs, communication can be either synchronous, asynchronous or a combination of both. In synchronous communication, a sender application sends a request to a receiver application and must wait for a reply before it can continue with its processing. Then, in asynchronous communication, a sender application sends a message to a receiver application and continues its processing before receiving a response. Asynchronous communication allows the loose coupling of applications, eliminating the need for connection management. This results in an applications integration solution that is more flexible, agile, and scalable essential attributes denoting what Industry 4.0 seeks for. For designing an integration solution, asynchronous communication offers a number of advantages over synchronous communication, especially when it comes to services in a Service Oriented Architecture. In synchronous communication patterns, timeouts are more common when an application has to wait for responses from several other applications. This means that the availability of services increases since individual processes are not blocked out as frequently due to waiting on other sub-processes to complete [17]. 
At present, a model-based integration approach is in its infancy and requires significant future research efforts. Many researchers agree that modelling from the CPS is a sizeable obstacle for companies that handle big data and obtain any profitable analysis for prediction. It has been suggested to tackle uncertainties within the data analysis. Tool integration and support from model-based systems and rapid construction of domain-specific tool chains are also suggested from present research [16].

\section{Virtual Prototyping with Computer-Automated Design}

Utilizing Evolutionary Computation, CAutoD accelerates and optimizes the tedious process of trial-and-error by reversing a design problem into a simulation problem, then automating such digital prototyping by intelligent search via biologicalinspired machine learning [18]. Experimental research in order to validate scientific results of the theoretical work is also what researchers suggest. Validation and implementation of this approaches help with a fast rhythm of acquiring knowledge and developments. What is trending now will not be the same in a few more years' time. When launching projects like smart manufacturing and Industry 4.0, companies should stay one step ahead and put efforts on innovative resources for advanced results.

\section{DISCUSSION AND CONCLUSION}

As stated so far, there exist challenges and future directions when tackling the subject of Industry 4.0, as argued in [3]. These include general reluctance to change by stakeholders, threat of redundancy of corporate IT departments and a lack of adequate skill-sets to expedite the march towards the 4th Industrial Revolution.

Many other trends have developed for Smart Manufacturing, not only in Germany with Industry 4.0, but also in the United States such as the Smart Manufacturing Leadership Coalition (SMLC). What SMLC presents is the infusion of intelligence that transforms the way industries conceptualize, design and operate the manufacturing enterprise [19]. Both perspectives agree on what challenges have to overcome in order to achieve what they pursue, such as analysis of big data-information, interoperability and scalability, among others.

So far, smart manufacturing approaches, analysis, virtualization and the new tendencies like the Industry 4.0 and big data studies have been studied. Summarizing the related work and developments leads to focus on the aspects facing Industry 4.0, such as methodologies that integrate collaborative systems. In this case, researchers suggest that a well-funded methodology that integrates CPS, cloud computing, virtual designs and real-time analysis is key to achieving innovation and a high productivity, because the system at the end becomes self-aware and self-predictive among other properties that are suitable for future research.

\section{REFERENCES}

[1] Lee, J., H.-A. Kao and S. Yang (2014). "Service Innovation and Smart Analytics for Industry 4.0 and Big Data Environment." Procedia CIRP 16(0): 3-8.)

[2] Lasi, H., P. Fettke, H.-G. Kemper, T. Feld and M. Hoffmann (2014). "Industry 4.0." Business \& Information Systems Engineering 6(2): 239-242.
[3] Kagermann, H., W. Wahlster and J. Helbig (2013). "Recommendations for implementing the strategic initiative INDUSTRIE 4.0." ACATECH NATIONAL ACADEMY OF SCIENCE AND ENGINEERING(3).R. Nicole, "Title of paper with only first word capitalized," J. Name Stand. Abbrev., in press.

[4] MacDougall, W. (2014). "INDUSTRIE 4.0 SMART MANUFACTURING FOR THE FUTURE." MECHANICAL \& ELECTRONIC TECHNOLOGIES, GERMANY TRADE \& INVEST(4): 40.

[5] Monostori, L. (2014). "Cyber-physical Production Systems: Roots, Expectations and R\&amp;D Challenges." Procedia CIRP 17(5): 9-13.

[6] Technology, N. I. o. S. a. (2013). "Strategic R\&D Oportunities for 21st Century Cyber-Physical Systems." Foundations for Innovation in Cyber-Physical Systems Workshop(6): 32.

[7] Sztipanovits, J., X. Koutsoukos, G. Karsai, N. Kottenstette, P. Antsaklis, V. Gupta, B. Goodwine, J. Baras and W. Shige (2012). "Toward a Science of Cyber\&\#x2013; Physical System Integration." Proceedings of the IEEE 100(7): 29-44.

[8] Lichen, L. (2015). Model Integration and Model Transformation Approach for Multi-Paradigm Cyber Physical System Development. Progress in Systems Engineering. H. Selvaraj, D. Zydek and G. Chmaj, Springer International Publishing. 330: 629-635.

[9] Sztipanovits, J., T. Bapty, S. Neema, L. Howard and E. Jackson (2014). OpenMETA: A Model- and Component-Based Design Tool Chain for Cyber-Physical Systems. From Programs to Systems. The Systems perspective in Computing. S. Bensalem, Y. Lakhneck and A. Legay, Springer Berlin Heidelberg. 8415: 235-248.

[10] Lattmann, Z., A. Nagel, J. Scott, K. Smyth, C. vanBuskirk, J. Porter, S. Neema, T. Bapty, J. Sztipanovits, J. Ceisel and D. Mavris (2012). Towards Automated Evaluation of Vehicle Dynamics in System-Level Designs. ASME 2012 International Design Engineering Technical Conferences and Computers and Information in Engineering Conference. A. S. M. ENGINEERS: 1131-1141.

[11] Eremenko, P. (2011). Philosophical Underpinnings of Adaptive Vehicle Make. DARPA-BAA-12-15. Appendix 1 (December 5, 2011), Arlington, VA.

[12] De Wolf, T. and T. Holvoet (2003). Towards autonomic computing: agent-based modelling, dynamical systems analysis, and decentralised control. Industrial Informatics, 2003. INDIN 2003. Proceedings. IEEE International Conference on.

[13] Jun, C., W. Xing, Z. Shilin, Z. Wu and N. Yanping (2012). A Decentralized Approach for Implementing Identity Management in Cloud Computing. Cloud and Green Computing (CGC), 2012 Second International Conference on.

[14] Boyd, S., N. Parikh, E. Chu, B. Peleato and J. Eckstein (2010). "Distributed Optimization and Statistical Learning via the Alternating Direction Method of Multipliers." Foundations and Trends ${ }^{\circledR}$ in Machine Learning 3(13): 1-122.

[15] Andreadis, G., G. Fourtounis and K.-D. Bouzakis (2015). "Collaborative design in the era of cloud computing." Advances in Engineering Software 81(11): 66-72.

[16] Givehchi, O., H. Trsek and J. Jasperneite (2013). Cloud computing for industrial automation systems- A comprehensive overview. Emerging Technologies \& Factory Automation (ETFA), 2013 IEEE 18th Conference on.

[17] Yoong, L., P. Roop, Z. Bhatti and M. Y. Kuo (2015). Introduction to Synchronous Programming Using Esterel. Model-Driven Design Using IEC 61499, Springer International Publishing: 35-64.

[18] Bukata, B. B. and L. Yun (2011). Reviewing DSTATCOM for smart distribution grid applications in solving power quality problems. Automation and Computing (ICAC), 2011 17th International Conference on.

[19] Swink, D. (2014). Smart Manufacturing Leadership Coalition (SMLC). OSI-SOFT, SAN LEANDRO, CA. 